\title{
Researches on the Relationship between KAP of Physical Exercise and Life Quality of Female College Students
}

\author{
Wang Maosong \\ Society and Law School, Shandong Women's University \\ Jinan, China, 250300
}

\begin{abstract}
Knowledge, attitude and practice(KAP) towards physical exercise are the influencing factors for College Students' active participation in daily exercises. With the random sampling method, 268 female students from Shandong Women's University were selected for the study. Through questionnaire, the knowledge, attitude and practice of female students in physical exercises were analyzed, life quality of female college students were measured by SF-36 scale, the correlation between KAP and life quality was analyzed. The total score of life quality of students was $(544.68 \pm 115.80)$. The correlation analysis showed that the quality of household registration and the total score of belief were correlated with the total score of life quality $(P<0.05)$. In this regard, in order to urge female college students to strengthen physical exercise and physical fitness, schools should strengthen guidance, especially pay attention to the differences among students from different household registration sources.
\end{abstract}

Keywords-female college students; physical exercise; KAP; life quality

\section{INTRODUCTION}

Physical fitness of college students is an important factor to guarantee their study and personal development. However, recent studies show that college students lack physical exercise and their physical fitness is worrying. In recent years, more and more Chinese scholars have paid attention to the problem of College Students' physical fitness. Bad physical fitness not only directly leads to the decline of physical fitness, affects students' study and daily life, but also reduces the social competitiveness of college students from a long-term perspective, which is not conducive to the cultivation of talents. Daily and regular physical exercise is the key to maintain health and ensure physical fitness. KAP theory model is one of the models to change human health-related behavior, and it is also a behavioral intervention theory. It divides the change of human behavior into three successive stages: acquiring knowledge, generating beliefs and forming behaviors. Generally speaking, the deeper the knowledge is, the stronger the tendency of implementation is. Knowledge is the basis of action and the source of strength. The same is true of physical exercise. Only when you have certain sports knowledge and belief, can you take action. In view of the situation of female college students' physical exercise, we designed and measured the KAP questionnaire of female college students' physical exercise. To evaluate the life quality of female college students, we choose SF-36 scale to measure it. SF-36 (36-item Short Form Health Survey) was developed from Boston Health Research in the United States on the basis of the Medical Outcomes Study-short from (MOSSF) developed by Stewartse in 1988. It can comprehensively reflect the health status of the population, simple items, easy to manage and operate, and can well assess the life quality of a certain population. It is one of the general scales for the evaluation of life quality. In this regard, we hope to investigate the current situation of College Students' physical exercise through questionnaires, and analyze the results of questionnaires on life quality. In this paper, 268 female college students from Shandong Women's College were selected by random sampling method. Through questionnaire, the KAP of female college students in physical exercise were analyzed, and the correlation with life quality was analyzed. In order to improve the life quality of female college students and improve their participation in physical exercise, this paper provides a reference.

\section{DATA AND METHODS}

\section{A. General data}

By means of random sampling, 268 female college students from Shandong Women's University were selected to conduct a questionnaire survey. A total of 268 questionnaires were distributed and 268 questionnaires were recovered, with a recovery rate of $100 \%$ and a validity rate of $100 \%$.

\section{B. Methods}

In the questionnaire survey, the KAP of physical exercise and the life quality were investigated. This part of the questionnaire includes general demographic characteristics (household registration, age, grade, specialty), physical exercise knowledge ( 7 items), physical exercise belief (5 items), and physical exercise behavior (5 items).Among them, the knowledge of physical exercise is given 0 and 2 points respectively according to the correct answers; the belief of physical exercise is divided into disagreement, disagreement, general, consent and agreement, giving 1, 2, 3, 4 and 5 points respectively. The evaluation of physical exercise behavior is specific for the number of weekly exercises, single exercise time, exercise plan and whether it is true or not. Plan and exercise.SF-36 scale made by Fang Jiqian of Sun Yat-sen University was selected for life quality evaluation. The scale was divided into eight dimensions: physiological function (RP), vitality (VT), physical pain (BP), social function (SF), mental health $(\mathrm{MH})$, general health $(\mathrm{GH})$, emotional function $(\mathrm{RE})$, and physiological function $(\mathrm{PF})$, with a full score of 1 in each dimension. The higher the score is, the better the life quality will be. Before the actual operation of the questionnaire, the consistency test and retest correlation analysis showed that the questionnaire had good reliability and could be used in this study. 


\section{Statistics}

SPSS 19.0 was used to analyze the data, the measurement data was expressed by (mean \pm standard), the counting data was expressed by rate, and the correlation analysis was tested by Person correlation. When $P<0.05$, the difference was statistically significant.

\section{RESULTS}

\section{A. General demographic characteristics data}

In terms of the nature of household registration, 268 female college students have 210 rural households, 58 urban households, and the proportion of rural households is $78.36 \%$. In terms of age, the average age of 268 female college students was $(19.99+0.96)$ years, and the highest proportion of 20 -year-olds was $44.03 \%$. In terms of grade distribution, freshmen and seniors are all distributed, with the highest proportion of sophomores being $64.43 \%$. In terms of majors, 268 female college students came from Literature, Management, Pedagogy, Art, Sociology, Economics, Computer Science and Law.

\section{B. KAP data of physical exercise}

The average score of physical exercise knowledge and belief of 268 female college students was $(10.16 \pm 1.98)$ and (18.68 \pm 2.54$)$. In terms of physical exercise behavior, $40.67 \%$ $(n=109)$ exercised 1-2 times a week, $24.25 \%(n=65)$ exercised 3-4 times a week, $21.27 \%(\mathrm{n}=57)$ did not exercise, $6.72 \%$ $(n=18)$ exercised 4-5 times a week, $7.09 \%(n=19)$ exercised every day, and $57.09 \%(n=153)$ exercised for 30 minutes at a single time. Among them, 37.31\% $(n=100)$ had 30-60 minutes, only $5.60 \%(n=15)$ had more than 60 minutes; $60.83 \%(n=163)$ had no corresponding plan, $39.18 \%(n=105)$ had formulated corresponding plan; and $8.58 \%(\mathrm{n}=23)$ had been exercising according to plan. In the way of physical exercise, the main ones are running $(46.27 \%, \mathrm{n}=124)$, walking $(28.73 \%, \mathrm{n}=77)$, $\operatorname{gym}(5.60 \%, \mathrm{n}=15)$.

\section{Life quality data}

In the evaluation of life quality, the total score of SF-36 was $(544.68 \pm 115.80)$. The specific scores of each dimension were: physiological function $(76.87 \pm 32.69)$, vitality (66.06 \pm 14.29$)$, physical pain $(40.75 \pm 33.32)$, social function (93.00 \pm 18.77$)$, mental health $(64.10 \pm 13.53)$, general health (69.64 \pm 17.02$)$, emotional function (60.82 \pm 38.53$)$, physiological function $(73.43 \pm 14.71)$. Compared with the SF-36 norm of Chinese female youth (18-25 years old), the four indicators of physiological function (RP), physiological function (PF), body pain (BP) and emotional function (RE) of female college students in this study are lower than the norm. Table 1 shows the differences between the SF-36 scores of female college students and the Chinese norm.
TABLE I COMPARISON OF SF-36 SCORES OF FEMALE COLLEGE STUDENTS WITH CHINESE NORMS

\begin{tabular}{lll}
\hline Item & Female College Students & Chinese Norm Score \\
\hline PF & $73.43 \pm 14.71$ & $90.2 \pm 11.6$ \\
RP & $76.87 \pm 32.69$ & $90.0 \pm 21.2$ \\
BP & $40.75 \pm 33.32$ & $84.6 \pm 18.1$ \\
GH & $69.64 \pm 17.02$ & $62.4 \pm 17.3$ \\
VT & $66.06 \pm 14.29$ & $54.0 \pm 18.8$ \\
SF & $93.00 \pm 18.77$ & $85.8 \pm 15.3$ \\
RE & $60.82 \pm 38.53$ & $85.9 \pm 28.5$ \\
MH & $64.10 \pm 13.53$ & $54.3 \pm 20.5$ \\
\hline
\end{tabular}

\section{Data of correlation analysis}

Through correlation analysis, it was found that the total score of life quality was related to the nature of household registration $(r=0.145, P=0.018)$, and the total score of belief $(r=0.151, P=0.013)$.

\section{DISCUSSION}

\section{A. Current situation of college students'physical exercise}

General Secretary Xi Jinping made clear demands on youth sports work many times. He pointed out that it is an important part of the great rejuvenation of the Chinese nation to make youth stronger than China and sports stronger than China. Previous studies have shown that there are many problems in the physical quality of College students. In the report on the development of Chinese youth sports (2015), it is pointed out that the physical dormitory of college students is worse than that of middle school students, and the endurance quality of college students continues to decline. The outline of the 13th Five-Year Plan clearly puts forward the implementation of the youth sports promotion plan, cultivating youth sports hobbies and sports skills, promoting and popularizing sports such as football, basketball, volleyball, ice and snow, and improving the youth physical health monitoring system. The Outline of the Healthy China 2030 Plan, which regards adolescents as the key group to improve the physical fitness of the whole people, puts forward specific goals and requirements for promoting adolescent sports activities. The National Fitness Program (2016-2020) takes teenagers as the key group to implement the National Fitness Program, vigorously popularizes teenagers' sports activities and improves their physical fitness. The Middle and Long Term Youth Development Plan (2016-2025) regards the youth physical health promotion project as a key project to encourage young people to take physical exercise and improve their physical fitness so as to make it a lifestyle and fashion for young people to adhere to physical exercise. In this regard, some scholars believe that the key to college students' physical fitness exercise lies in the change of consciousness. At present, the main physical exercise items in Colleges and universities are mainly physical education courses. Students only care about performance points and do not really participate in physical exercise, not to mention the national fitness. In western universities, physical exercise is mainly in gyms and related clubs, not related to academic performance, and students' participation is higher. In the physical exercises of female college students in our university, the main methods are running and walking. The proportion of doing exercises in gymnasium and other clubs is only $5.60 \%$. $57.09 \%$ of the time for a single physical exercise is within 30 minutes. Although students choose more ways of doing physical exercises, they still concentrate on traditional sports. It 
is still necessary to promote the atmosphere of students' physical exercise and enhance their awareness of physical exercise.

\section{B. Significance of life quality data}

This paper studies the correlation between knowledge, belief and behavior of physical exercise and life quality of 268 female college students. In terms of knowledge, belief and behavior of physical exercise, knowledge $(10.16 \pm 1.98)$ and belief $(18.68 \pm 2.54)$ have little overall dispersion, which indicates that the knowledge and belief of female college students in physical exercise are relatively uniform. Compared with the SF-36 norm of Chinese young women (18-25 years old), the four indicators of physiological function (RP), physiological function (PF), physical pain (BP) and emotional function (RE) of female college students in this study are lower than the norm. Lower than the norm of physiological function (RP), physiological function (PF) indicators, which to a certain extent reflects the female college students because of physiological reasons have some impact on life and learning, and these effects should be sustained. Physical pain (BP) may be due to physiological reasons or physical deterioration. Emotional function (RE) scores indicate that female college students' emotions are relatively fragile. Some female college students may be affected by their emotional life, which will be further investigated in the next study. Considering the gender characteristics of female college students, it is understandable that the four indicators of physiological function (RP), physiological function (PF), body pain (BP) and emotional function (RE) are lower than the norm. Therefore, relevant education should be strengthened to promote students' understanding of emotional and physical functions, enhance their adaptability and coping ability, so as to ensure the study and daily life of female college students.

\section{Significance of data for correlation analysis}

In the correlation analysis, the correlation between the total score of life quality and the total score of household registration $(r=0.145, P=0.018)$ and belief $(r=0.151, P=$ 0.013) was significant. In terms of the nature of household registration, this study is divided into urban household registration and rural household registration. Relevant analysis shows that the total score of female college students' life quality in rural household registration is relatively higher than that in urban household registration. The female college students' life quality in rural household registration is better than that in urban household registration. The proportion of female college students in rural household registration in this study is $78.36 \%$. The situation of students' residence registration in our school is basically the same, which indicates that more attention should be paid to the life quality of female college students with urban residence registration. In this regard, we usually subjectively think that female college students with urban household registration are better than those with rural household registration in terms of living conditions, mainly because the students with two households spend different money. Therefore, in theory, we may think that college students with urban hukou will pay more attention to the life quality, and their SF-36 score should be higher than that of female students with rural hukou. However, this study shows that the total score of life quality of female college students with rural household registration is relatively higher. For this reason, we consider that female college students with rural household registration in the study sample pay more attention to physical fitness exercise, while female college students with urban household registration may reduce their attention to physical fitness because of more abundant social relations. The reasons should be studied in depth.

In the evaluation index of belief, there are five items in this paper. The results show that the higher the belief score, the better the life quality score of female college students, indicating the importance of physical exercise attitude on the life quality. Further analysis found that in the correlation analysis of beliefs, weekly exercise frequency, specialty, single exercise time, exercise plan, exercise mode, emotional function, physiological function, social function, total score of life quality were correlated with them $(P<0.05)$. Among them, weekly exercise frequency, single exercise time, exercise plan, and total score of life quality were correlated with them $(P<$ $0.05)$. Exercise methods are all related items of physical exercise behavior, indicating the importance of belief influencing behavior, while emotional function, physiological function and social function are all components of the total score of life quality. This shows that attitude to physical exercise can affect the physiological, emotional and social functions of female college students, and attitude to physical exercise can affect the life quality. The impact is multifaceted.

\section{CONCLUSION}

The correlation between KAP of physical exercise and life quality of female college students is mainly the belief, belief or attitude of physical exercise, which indicates that the attitude of female college students in physical exercise needs further guidance. In this regard, schools should strengthen the construction of fitness stadiums, enrich sports-related associations and clubs, and increase various sports competitions to enhance the atmosphere and awareness of female college students' physical exercise.

\section{ACKNOWLEDGMENT}

This research was financially supported by Special Project of the Key Research Base of Youth Quality Education of Shandong Province (Project No. 18SZJ025).

\section{REFERENCES}

[1] Song Haiyan, Li Zhifang, Yang Yang, et al. The relationship between college students' knowledge, belief and behavior in physical exercise and life quality [J]. School Health, 2010, 31 (11): 1321-1322. (In Chinese)

[2] Hao Yuantao, Fang Jiqian. Introduction to the Chinese version of the World Health Organization life quality Scale and instructions for its use [J]. China Tissue Engineering Research, 2000, 4 (8): 1127-1129. (In Chinese)

[3] Wang Xiaofeng, Wang Xiangquan. Analysis of the change of College Students' physical fitness and its causes [J]. Journal of Demography, 2018, 40 (2): 86-95. (In Chinese)

[4] Zhu Haitao, Yang Shun, Zhang Yong, et al. Investigation and analysis of the current situation of College Students' health consciousness and physical health-Taking Chongqing as an example [J]. Journal of Guangzhou Institute of Physical Education, 2019, 39 (1): 27-30. (In Chinese)

[5] Yang Yun. The realistic predicament of College Students' physical health crisis and the way to alleviate it: reflections on the practice of College Public Physical Education Teaching [J]. Research on educational development, 2018, 38 (5): 29-34. (In Chinese)

[6] Lu Juxian, Liu Shuduan. A comparative study of physical exercise in school physical education reform between China and the United States 
[J]. Contemporary sports science and technology, 2018,8(1): 129-131. (In Chinese)

[7] Wang Hongmei, Li Lu, Shen Yi. The Chinese SF-36 scale was used to study the life quality of urban residents in Hangzhou [J]. Chinese Journal of Preventive Medicine, 2001, 35 (6): 428-430. (In Chinese)

[8] Wang Ruming. Study on Optimizing the Operating Mechanism of Sports Societies to Encourage Students' Self-Fitness [J]. Youth Sports, 2016 (8): 19-20. (In Chinese)
[9] Zhang Jianyu. Research on the integrated club-based teaching mode of physical education in and out of class under the credit system [J]. Journal of Guangzhou Institute of Physical Education, 2018, 38 (1): 121-124. (In Chinese)

[10] Xue Bing, Zhao Ye. Research on the Connotative Promotion Path of College Students' Three Walks Activities: Taking the Sports and Fitness Association of Southwest Jiaotong University as an example [J]. Modern Communication, 2018, 489 (19): 131-132. (In Chinese) 